Original paper

\title{
Change in $\gamma$-glutamyl transpeptidase activity as a useful tool in identifying a group of patients with elevated risk of hepatocellular carcinoma development after DAA treatment of chronic hepatitis C
}

Dorota Orzechowska', Katarzyna Klimowicz', Anna Stępień1, Tomasz Mikuła², Mariusz Sapuła², Alicja Wiercińska-Drapało²

'Department of Internal Medicine and Hepatology, Central Clinical Hospital of Ministry of the Interior and Administration, Warsaw, Poland 2Department of Infectious, Tropical Diseases and Hepatology, Medical University of Warsaw, Warsaw, Poland

\begin{abstract}
Aim of the study: Hepatitis C virus (HCV)-related hepatocellular carcinoma (HCC) incidence will be diminishing due to use of direct acting antiviral agents (DAA), but there is still constant risk for HCC development. Elevated serum $\gamma$-glutamyl transpeptidase (GGT) activity is associated with increased risk of liver cancer. In our study we tried to determine whether change in GGT activity may be useful in identifying patients with elevated risk of HCC development after DAA treatment.

Material and methods: The study population consisted of 111 patients with chronic hepatitis $\mathrm{C}(\mathrm{CHC})$ treated with DAA. Laboratory tests [alanine aminotransferase (ALT), GGT, $\alpha$-fetoprotein (AFP)] and liver stiffness measurement (using FibroScan) were performed at the beginning and at the end of therapy.

Results: Pre-treatment ALT activity, GGT activity and AFP concentration in patients with CHC were directly associated with the stage of liver fibrosis. Elimination of HCV after DAA treatment caused significant reduction in serum GGT activity and was not associated with pre-treatment liver fibrosis. AFP concentration was significantly lower after treatment. It was observed regardless of pre-treatment AFP concentration, but the largest reduction was demonstrated in the group of patients with advanced fibrosis. In multivariate analysis there was no significant difference in GGT activity after treatment only in patients with pre-treatment normal AFP concentration and advanced liver fibrosis.

Conclusions: Patients who after achieving a sustained virological response (SVR) did not lower both AFP concentration and GGT activity may have higher risk of HCC development. Special monitoring may be required in patients with advanced liver fibrosis and normal AFP concentration before treatment.
\end{abstract}

Key words: hepatocellular carcinoma, hepatitis C virus, $\alpha$-fetoprotein, $\gamma$-glutamyl transpeptidase.

Address for correspondence:

Dorota Orzechowska, Department of Internal Medicine and Hepatology, Central Clinical Hospital of Ministry of the Interior and Administration, Warsaw, Poland, e-mail: dorota.orzechowska@onet.eu

\section{Introduction}

Hepatitis $\mathrm{C}$ virus (HCV) infection is a significant epidemiological problem. According to World Health Organization (WHO) data, it is estimated that more than 71 million people in the world are infected with HCV [1], of which around 7 million are in Europe [2]. Epidemiological studies conducted in Poland revealed that $\mathrm{HCV}$ infection occurs in $0.5 \%$ of the population, which is about 150,000 adult inhabitants [3]. 
Although the virus has been known since 1989, it is still one of the main causes of mortality associated with liver diseases. In the natural course of HCV infection, $20-30 \%$ of infected patients will suffer from liver cirrhosis after 20-30 years of infection [4]. Furthermore, each year approximately $1-8 \%$ of those cirrhotic patients will develop hepatocellular carcinoma (HCC). The risk of HCC development is about 15-20 times higher in patients with HCV infection compared with the healthy population [5]. Prognosis in HCC is one of the worst among all cancers [6]. Worldwide, HCC is the second leading cause of cancer death in men and the sixth in women. HCV infection is largely responsible for the overall increase in HCC incidence in developed countries in recent years [7].

Successful antiviral therapy plays a crucial role in the prophylaxis of $\mathrm{HCC}$ in $\mathrm{HCV}$ infected patients. After successful treatment, the risk for HCC development is much lower, although it does not disappear completely $[5,8,9]$. Patients who achieved a sustained virological response (SVR) also have a lower overall mortality rate, reduction of fibrosis and fewer extrahepatic manifestations. Current therapeutic strategies guarantee over $90 \%$ rate of SVR, regardless of pre-treatment liver fibrosis or HCV genotype. Due to extensive use of direct acting antiviral agents (DAA), we can expect that HCV-related HCC incidence will be diminishing in the following years, but there is still constant risk for HCC development even 10 years after antiviral treatment $[5,9,10]$.

The most popular marker for HCC is $\alpha$-fetoprotein (AFP). Elevated AFP concentration is observed in chronic viral hepatitis, liver cirrhosis, primary biliary cholangitis, obstructive jaundice and alcohol-related liver disease [11]. It is suspected that elevated AFP concentration in those diseases is an effect of inflammation, necrosis and damage of hepatocytes [12]. The highest correlation is observed in HCV infection, probably because viral core proteins cause activation of AFP production through activation of transcription.

Reduction in AFP concentration in patients with chronic hepatitis $\mathrm{C}$ (CHC) was observed after IFNbased treatment $[5,12]$. It has been established that HCC is statistically more frequent in patients who after successful IFN-based therapy did not achieve low AFP values $(<20$ or $<5 \mathrm{ng} / \mathrm{ml})[13,14]$. In patients who did not achieve SVR, but had a low level of AFP after treatment $(<10 \mathrm{ng} / \mathrm{ml})$, the risk for HCC development was significantly reduced, irrespectively of pre-treatment fibrosis [15].

In patients with advanced liver fibrosis, higher activity of $\gamma$-glutamyl transpeptidase (GGT) was found [16]. Many previous studies proved that higher activity of GGT before treatment was linked to lower probability of SVR [17-20]. Furthermore, elevated GGT before treatment, even in non-cirrhotic patients who achieved SVR after IFN + RBV-based regimen, was linked to higher risk of HCC development [21].

$\gamma$-glutamyl transpeptidase is an enzyme located on outer surface of the cell membrane, present in nearly every cell, especially in the liver. It catalyses transfer of gamma-glutamyl groups from glutathione to amino acids and dipeptides, ensures continuous cysteine supply in cells and intracellular synthesis of glutathione. Glutathione is the most powerful, most common, most universal and most important intracellular antioxidant. It is proven that serum GGT activity is directly proportional to the level of oxidative stress [22]. Elevated serum GGT activity is associated with increased risk of cardiovascular complications, diabetes, arterial hypertension, as well as increased overall mortality and elevated risk of cancer, especially prostate, breast and liver cancer [23]. GGT is commonly used in diagnostics of liver diseases, mainly alcohol-related liver disease and cholestatic liver diseases. However, this parameter is not routinely measured in patients with $\mathrm{CHC}$.

Patients with advanced liver fibrosis treated for $\mathrm{HCV}$ infection should be still regularly screened for HCC development. Abundant scientific evidence confirms the favourable role of SVR in inhibiting the progression of cirrhosis and therefore diminishing the incidence of HCC. With the introduction of DAA-based regimens, the index of SVR is significantly higher. As the population of patients achieving SVR will grow, it is important to identify patients at higher risk for HCC development. Besides known risk factors such as liver cirrhosis, age, and ethanol consumption, further studies are needed to identify more sensitive risk factors and biomarkers for HCC. The role of AFP as a basic screening strategy is insufficient [5].

In our study we tried to determine whether a change in GGT activity may be useful in identifying a group of patients with elevated risk of HCC development after DAA treatment.

\section{Material and methods}

The study population consisted of 111 patients infected with HCV, treated with DAA during 2015-2016 in the Department of Infectious, Tropical Diseases and Hepatology of Medical University of Warsaw and in the Department of Internal Medicine and Hepatology of the Central Clinical Hospital of the Ministry of the Interior and Administration in Warsaw. Characteristics of the study population are shown in Table 1. 
The study was conducted in accordance with a Polish therapeutic programme funded by the National Health Fund [24]. Adult patients, with detectable HCV RNA, who fulfilled therapeutic programme criteria (i.e. HCV genotype 1 or 4 , anti-HCV positive, confirmation of liver fibrosis either in liver histopathology or in elastography) and underwent DAA treatment in study centres, were included in the study. Exclusion criteria were the same as those listed in the therapeutic programme, i.e. absent HCV RNA in serum before treatment, decompensated cirrhosis Child-Pugh C, alcohol and/or drug dependence, pregnancy or breastfeeding [24]. Additional exclusion criteria were lack of SVR and development of HCC during antiviral treatment.

Two patients were excluded from the analysis. One of them developed HCC during antiviral treatment, and the other did not achieve SVR.

Not all tests were performed in the whole study group due to lack of compliance and few laboratory errors (haemolysis, lack of diagnostic material).

Laboratory tests [alanine aminotransferase (ALT), GGT, AFP] and liver stiffness measurement (using FibroScan) were performed at the beginning and at the end of antiviral treatment.

Alanine aminotransferase activity was measured with the photometric method using the COBAS test on the day of the beginning and at the end of DAA treatment, i.e. after 12 weeks and in 2 cases after 8 weeks. The assessment was done in 108 patients. The normal range was 5-41 U/l.

$\alpha$-fetoprotein concentration was measured with electrochemiluminescence (ECLIA) using the COBAS test on the day of the beginning and at the end of DAA treatment, i.e. after 12 weeks, in 2 cases after 24 weeks and in another 2 cases after 8 weeks. The assessment was done in 96 patients. The upper limit of normal was $8 \mathrm{ng} / \mathrm{ml}$.

$\gamma$-glutamyl transpeptidase activity was measured with the enzymatic-colorimetric method using the COBAS test on the day of the beginning and at the end of DAA treatment, i.e. after 12 weeks and in 2 cases after 8 weeks. The assessment was done in 79 patients. The normal range was 8-61 U/l.

Liver fibrosis was assessed in all patients $(n=109)$. Some of the patients had previously had liver biopsy $(n=66)$ with histopathological assessment of fibrosis using the METAVIR scale. We assessed liver fibrosis with non-invasive elastography using FibroScan in almost all patients $(n=105)$ on the day of the beginning of DAA treatment. The cut-offs were as follows: F0: $<5 \mathrm{kPa}, \mathrm{F} 1: 5.1-7.0 \mathrm{kPa}, \mathrm{F} 2: 7.1-9.5 \mathrm{kPa}, \mathrm{F} 3: 9.6-12.5 \mathrm{kPa}$, $\mathrm{F} 4: \geq 12.5 \mathrm{kPa}$. Stage of fibrosis assessed in liver biopsies was consistent with the results of elastography. We
Table 1. Study group characteristics

\begin{tabular}{|c|c|}
\hline Variable & \\
\hline \multicolumn{2}{|l|}{ Sex, $n(\%)$} \\
\hline Women & $49(44)$ \\
\hline Men & $62(56)$ \\
\hline Median age (years) & $58(41-63)$ \\
\hline Median BMI $\left(\mathrm{kg} / \mathrm{m}^{2}\right)$ & $26.4(24.2-29.3)$ \\
\hline \multicolumn{2}{|l|}{$\mathrm{BMI}, n(\%)$} \\
\hline$\geqslant 30$ & $19(17)$ \\
\hline $25-30$ & $55(49.5)$ \\
\hline$<25$ & $35(31.5)$ \\
\hline Lack of data & $2(2)$ \\
\hline \multicolumn{2}{|l|}{ Degree of liver fibrosis, $n(\%)$} \\
\hline $\mathrm{FO}-2$ & $82(74)$ \\
\hline F3-4 & $29(26)$ \\
\hline Median albumin concentration ( $\mathrm{g} / \mathrm{dl}$ ) & $3.76(3.45-4.03)$ \\
\hline Median platelet count $(\mathrm{k} / \mu \mathrm{l})$ & $197(150-247)$ \\
\hline Median HCV viral load (IU/ml) & $\begin{array}{c}1,010,000 \\
(417,500-2,105,000)\end{array}$ \\
\hline \multicolumn{2}{|l|}{ Genotype, $n(\%)$} \\
\hline 1 & $108(97)$ \\
\hline 4 & $4(3)$ \\
\hline \multicolumn{2}{|l|}{ Number of previous antiviral therapies, $n(\%)$} \\
\hline$\underline{0}$ & $53(48)$ \\
\hline 1 & $26(23)$ \\
\hline 2 & $17(15)$ \\
\hline 3 & $7(6)$ \\
\hline Unknown, but > 1 & $8(7)$ \\
\hline \multicolumn{2}{|l|}{ Duration of DAA treatment, $n(\%)$} \\
\hline 8 weeks & $2(2)$ \\
\hline 12 weeks & $107(96)$ \\
\hline 24 weeks & $2(2)$ \\
\hline \multicolumn{2}{|l|}{ Current treatment, $n(\%)$} \\
\hline Sofosbuvir and ledipasvir & $13(12)$ \\
\hline Sofosbuvir and ledipasvir with ribavirin & $17(15)$ \\
\hline Dasabuvir, ombitasvir, paritaprevir and ritonavir & $53(48)$ \\
\hline $\begin{array}{l}\text { Dasabuvir, ombitasvir, paritaprevir and ritonavir } \\
\text { with ribavirin }\end{array}$ & $25(22)$ \\
\hline Dasabuvir with ribavirin & $3(3)$ \\
\hline
\end{tabular}

BMI - body mass index, non-parametric variables presented as frequency (percentages); skewed variables presented as median (25-75 percentile).

repeated FibroScan in 101 patients at the end of DAA treatment.

All patients had undetectable HCV RNA at the end of treatment and achieved SVR. 
Statistical analysis was conducted with the statistical program Statistica 13.1 (StatSoft Poland). Distribution of studied parameters differed from normal; therefore to describe the study group, the medians and interquartile ranges were used. Qualitative variables were presented as percentages. To compare groups, the non-parametric Mann-Whitney $U$ test was used. Wilcoxon's character pair test was used for related variables. Due to violation of the assumptions of sphericity,

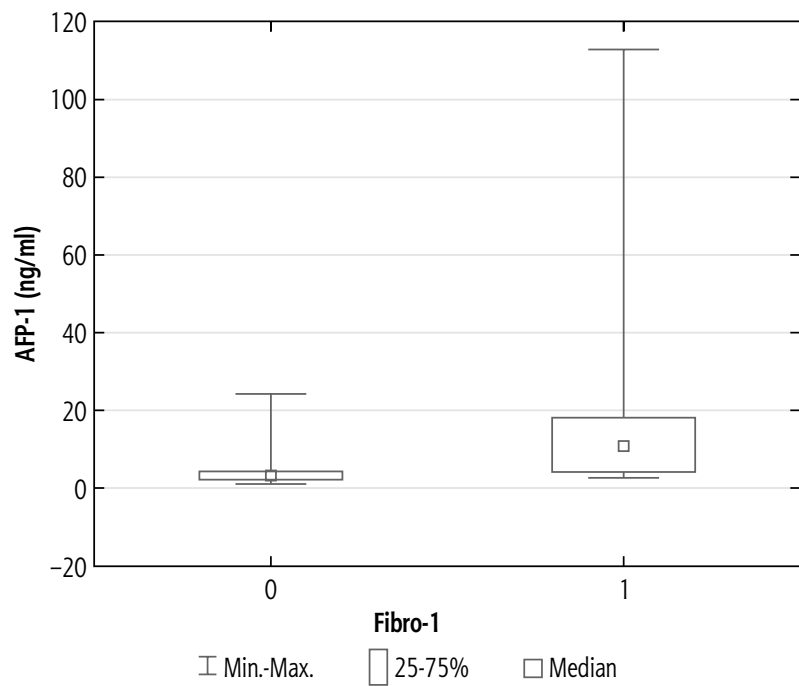

Fig. 1. $\alpha$-fetoprotein (AFP) concentration depending on stage of hepatic fibrosis pre-treatment

AFP-1 pre-treatment AFP concentration, fibro-1 - pre-treatment stage of liver fibrosis $(0=F 0-F 2,1=F 3-F 4)$

Table 2. Differences in liver stiffness, alanine aminotransferase (ALT), and $\gamma$-glutamyl transpeptidase (GGT) in groups with pre-treatment normal and elevated $\alpha$-fetoprotein (AFP) concentration

\begin{tabular}{lcc}
\hline & $\begin{array}{c}\text { Pre-treatment normal } \\
\text { AFP concentration } \\
(\boldsymbol{n}=79)\end{array}$ & $\begin{array}{c}\text { Pre-treatment } \\
\text { elevated AFP } \\
\text { concentration } \\
(\boldsymbol{n}=22)\end{array}$ \\
\hline Pre-treatment & & \\
\hline Liver stiffness $(\mathrm{kPa})$ & $5.80(4.45-8.40)$ & $17.30(11.90-31.60)$ \\
\hline ALT $(\mathrm{U} / \mathrm{l})$ & $47.00(32.00-62.00)$ & $106.50(73.00-123.00)$ \\
\hline $\mathrm{GGT}(\mathrm{U} / \mathrm{l})$ & $32.00(21.00-59.00)$ & $88.00(66.00-146.00)$ \\
\hline
\end{tabular}

Variables are presented as median (25-75 percentile). normality of distribution and homogeneity of variance, multivariate analysis of variance (MONOVA) was used to analyse changes over time. The Pillai-Bartlett trace was used as the test statistic. The Tukey test was used as the post-hoc test for one-dimensional multiple comparisons.

\section{Results}

Pre-treatment ALT $(p<0.001)$ and GGT activities $(p<0.05)$ in patients with CHC treated with DAA were positively associated with the stage of liver fibrosis. Pre-treatment AFP concentration was normal in subgroups with non-advanced fibrosis and increased along with stage of hepatic fibrosis $(p<0.001)$, which is demonstrated in Figure 1.

Patients with elevated pre-treatment AFP concentration had significantly up-regulated ALT and GGT activities ( $p<0.001, p<0.001$ respectively) and higher liver stiffness $(p<0.001)$ compared to those with normal AFP concentration before therapy. These data are presented in Table 2 .

It was confirmed in multivariate analysis that pre-treatment GGT activity was significantly higher in the group of patients with pre-treatment elevated AFP and advanced fibrosis compared to the group of patients with normal AFP and non-advanced fibrosis.

Elimination of HCV infection after DAA treatment caused a significant reduction in serum GGT activity $(p<0.001)$. GGT activity pre-treatment was $42 \mathrm{U} / 1$ and post-treatment $16 \mathrm{U} / 1$.

A significant decrease in GGT activity was observed in the group of patients with pre-treatment elevated as well as normal ALT activity ( $p<0.001$ in both groups). Both patients with pre-treatment elevated and normal ALT activity had significantly lower AFP concentration after treatment ( $p<0.001$ in both groups). A significant decrease in liver stiffness $(p<0.001)$ was observed in the group of patients with elevated ALT activity.

Changes in GGT activity, AFP concentration and liver stiffness after antiviral therapy in groups with pre-treatment normal and elevated ALT activity are presented in Table 3.

Table 3. Change in $\gamma$-glutamyl transpeptidase (GGT), $\alpha$-fetoprotein (AFP) and liver stiffness after direct acting antiviral agents (DAA) therapy in groups with pretreatment normal and elevated alanine aminotransferase (ALT)

\begin{tabular}{lcccc}
\hline \multirow{2}{*}{ Parameter } & \multicolumn{2}{c}{ Pre-treatment normal ALT $(\boldsymbol{n}=\mathbf{3 1})$} & \multicolumn{2}{c}{ Pre-treatment elevated ALT $(\boldsymbol{n}=\mathbf{7 8})$} \\
\cline { 2 - 5 } & Pre-treatment & Post-treatment & Pre-treatment & Post-treatment \\
\hline GGT $(\mathrm{U} / \mathrm{l})$ & $22.50(15.00-30.00)$ & $13.50(10.50-16.50)$ & $54.50(34.50-108.50)$ & $19.00(15.00-29.00)$ \\
\hline AFP $(\mathrm{ng} / \mathrm{ml})$ & $2.20(1.70-4.00)$ & $1.90(1.20-3.30)$ & $4.20(3.05-11.95)$ & $3.32(2.15-5.00)$ \\
\hline Liver stiffness $(\mathrm{kPa})$ & $4.90(4.10-7.30)$ & $4.90(4.50-6.10)$ & $8.40(5.40-17.10)$ & $6.80(4.90-14.50)$ \\
\hline
\end{tabular}

Variables are presented as median (25-75 percentile). 


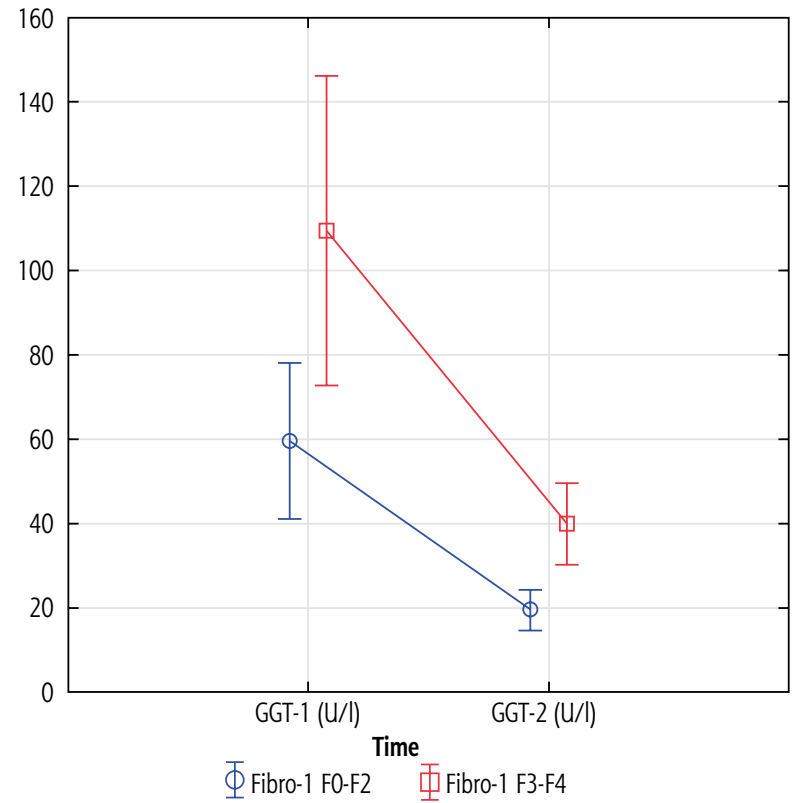

Fig. 2. Change in $\gamma$-glutamyl transpeptidase (GGT) activity after treatment depending on pre-treatment stage of liver fibrosis

GGT-1 - pre-treatment GGT activity, GGT-2 - post-treatment GGT activity, fibro-1 - pretreatment liver fibrosis

A significant decrease in GGT activity after DAA therapy was observed in almost all patients and was not associated with pre-treatment liver fibrosis. This correlation is presented in Figure 2.

AFP concentration was significantly lower after DAA treatment. AFP concentration pre-treatment was $3.70 \mathrm{ng} / \mathrm{ml}$ and post-treatment $3.00 \mathrm{ng} / \mathrm{ml}(p<0.001)$. Reduction in AFP concentration at the end of treatment was observed in the group of patients with normal as well as elevated AFP concentration before treatment.

The largest reduction in AFP concentration was noted in the group of patients with advanced fibrosis. These data are presented in Figure 3.

Additionally in the subgroup of patients with pre-treatment elevated AFP concentration, liver stiffness was significantly lower after DAA treatment $(p<0.05)$. These data are shown in Figure 4.

Furthermore, multivariate analysis, taking into consideration change in GGT activity after treatment, depending on pre-treatment AFP concentration and liver fibrosis was performed. Significant reduction in GGT activity after treatment was confirmed in three subgroups of patients: 1) with normal AFP concentration and non-advanced liver fibrosis pre-treatment; 2) with elevated AFP concentration and non-advanced liver fibrosis pre-treatment; 3 ) with elevated AFP concentration and advanced liver fibrosis before treatment. Only in the subgroup of patients with normal AFP concentration and advanced liver fibrosis before treatment

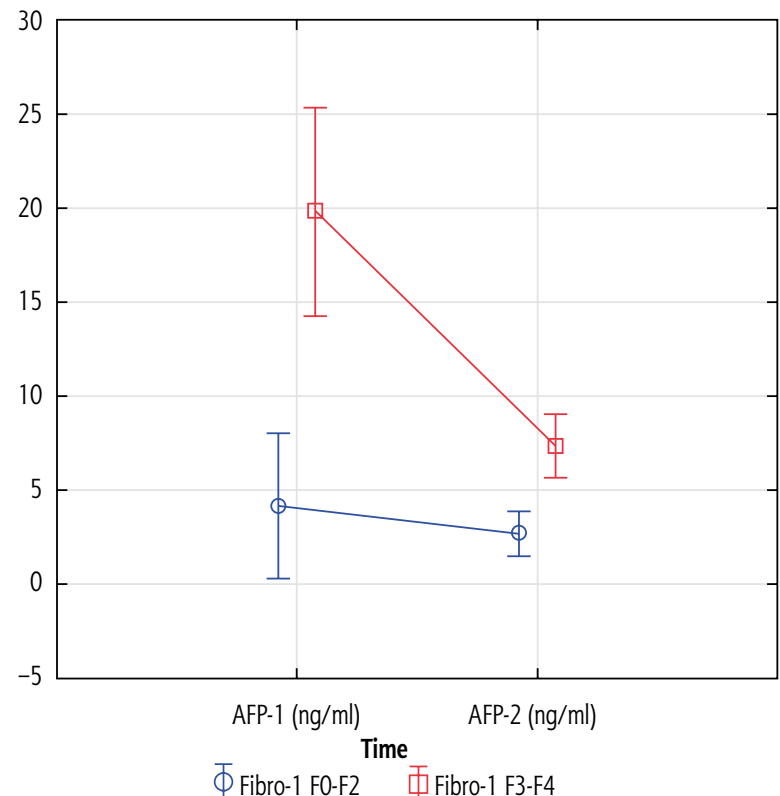

Fig. 3. Change in $\alpha$-fetoprotein (AFP) concentration after treatment depending on pre-treatment stage of liver fibrosis

AFP-1 - pre-treatment AFP concentration, AFP-2 - post-treatment ASP concentration, fibro-1 - pre-treatment stage of liver fibrosis

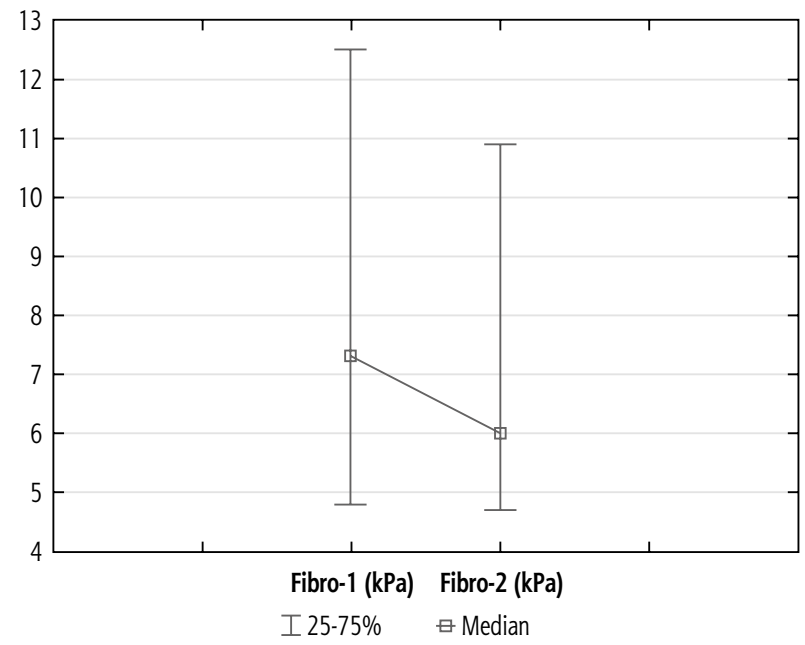

Fig. 4. Reduction of liver stiffness after direct acting antiviral agents (DAA) treatment in patients with pre-treatment elevated AFP concentration

Fibro-1 - pre-treatment liver stiffness, Fibro-2 - post-treatment liver stiffness

was there no significant difference in GGT activity after treatment. These data are presented in Figure 5.

\section{Discussion}

Elimination of HCV infection leads to reduction of the degree of inflammation and stage of liver fibrosis. Therefore the risk of development of liver cirrhosis and HCC is diminished. GGT is widely used in diagnostics of liver disease. Clinical implications of GGT activity 


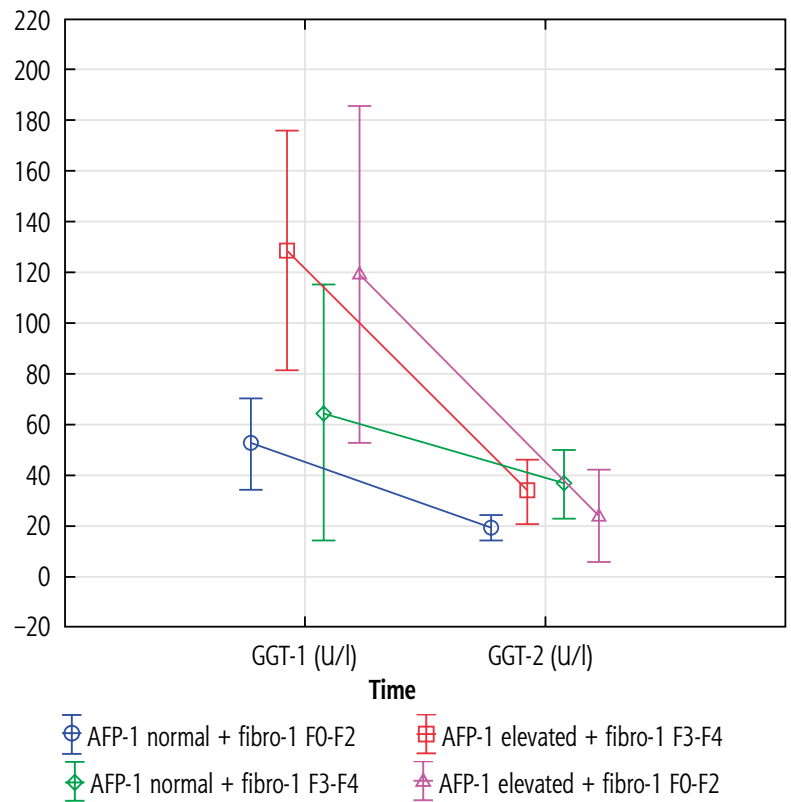

Fig. 5. Reduction of $\gamma$-glutamyl transpeptidase (GGT) activity after direct acting antiviral agents (DAA) treatment depending on pre-treatment $\alpha$-fetoprotein (AFP) concentration and stage of liver fibrosis

GGT-1 - pre-treatment GGT activity, GGT-2 - post-treatment GGT activity, AFP-1 - pretreatment AFP concentration, fibro-1 - pre-treatment stage of liver fibrosis

are still being underestimated, in spite of abundant research on the role of GGT in neoplasms.

In our study, a positive correlation between pre-treatment GGT and ALT activity and stage of hepatic fibrosis was demonstrated. Those observations are in concordance with the results of the study of Silva et al. They examined over 200 liver biopsies of HCV-infected patients and proved that GGT activity was elevated in a group of patients with advanced fibrosis. They suggested that GGT activity may be a biochemical marker of the stage of liver fibrosis [16]. In our study, we observed that a significant decrease in liver stiffness was observed only in the group of patients with elevated ALT activity before treatment. However, the pre-treatment result of elastography may be falsely overestimated due to elevated ALT activity. Therefore GGT activity may be a more useful biochemical parameter for assessment of liver fibrosis. Reduction in GGT activity after HCV RNA elimination may be an indicator of inhibition of fibrogenesis.

Elevated GGT activity was considered an unfavourable prognostic factor for achieving SVR with IFN-based regimens [17-20]. This correlation was not observed in our study. All patients treated with DAA achieved SVR, irrespectively of pre-treatment GGT activity. It probably results from different mechanisms of action of those drugs.

In our study a significant decrease in GGT activity after DAA treatment was observed in almost all $\mathrm{HCV}$ infected patients. GGT activity was significantly lower after treatment, both in groups of patients with elevated and normal ALT activity before treatment, as well as in groups of patients with both non-advanced and advanced liver fibrosis before treatment. Up to now, GGT activity has been assessed only in patients treated with DAA after liver transplantation, demonstrating an important reduction in GGT after achieving SVR [25].

It was proven in our study that the pre-treatment stage of liver fibrosis did not influence reduction of GGT activity after treatment. It may be expected that HCV RNA elimination is crucial for change of GGT activity. A similar observation was made in the HALT-C study, which included patients treated with low-dose IFN [17]. Everhart et al. demonstrated that higher GGT activity was connected with lower rate of SVR, more advanced liver disease and higher risk for HCC development. In this study, change in the stage of hepatic fibrosis was not linked to change in GGT activity. The authors suggest that GGT may be a marker of activity of the disease and not only of its severity.

Statistically, HCC develops more frequently in patients who do not have normalized AFP concentration after successful IFN-based therapy [13, 14]. Studies published on large groups of patients on DAA therapy proved that pre-treatment elevated AFP concentration is an important risk factor for HCC, even after elimination of HCV RNA [26, 27]. Up to now, only limited research on the reduction of AFP concentration after DAA treatment has been published [28]. In our study, pre-treatment AFP concentration was directly related to the stage of liver fibrosis. AFP concentration after DAA treatment was significantly lower, both in subgroups with elevated and normal ALT activity before treatment as well as in subgroups with non-advanced and advanced liver fibrosis before treatment. The largest decrease in AFP concentration after treatment was observed in patients with advanced liver fibrosis.

In our study, patients who at the beginning of treatment had elevated AFP concentration also had higher ALT and GGT activity and liver stiffness. Yang et al., in their study on about $300 \mathrm{HCV}$-infected patients, also observed a positive correlation between ALT activity and AFP concentration [29]. Furthermore, in our study, there was a clear correlation between GGT activity and AFP concentration before treatment. As long as ALT is a biochemical marker of the inflammatory activity, GGT may be an indicator of increased risk of carcinogenesis, inter alia through oxidative stress. In the study of Carr et al. on patients with HCC, it was demonstrated that simultaneous analysis of both GGT and AFP may be a useful tool in the diagnostics of small HCC [30]. Fluctuation of those parameters, 
even within the normal range, should prompt oncological surveillance.

In a multivariate analysis, patients with non-advanced liver fibrosis before treatment, independently of AFP concentration, had a significant decrease in GGT activity after DAA treatment. In the subgroup of patients with advanced liver fibrosis and elevated AFP concentration before treatment there was also significant reduction in GGT activity. This confirms the additional advantage of antiviral therapy in patients with advanced liver disease. However, in the subgroup of patients with advanced liver fibrosis and normal AFP concentration before treatment, there was no significant reduction in GGT activity after treatment. It may suggest permanence of a high level of oxidative stress. Considering the above stated conclusions, it can be assumed that the risk of HCC development is elevated in the subgroup of patients with normal AFP concentration and advanced liver fibrosis before treatment, who achieved SVR, and who did not achieve a significant decrease in GGT activity. This group of patients requires close oncological surveillance, especially because a normal AFP concentration may entail lower vigilance.

The reduction in GGT activity after DAA treatment reflects not only reduction of inflammatory activity and liver stiffness, but may also describe not yet identified cellular mechanisms leading to neoplastic transformation.

Our study has several limitations. First of all, the study did not include information on possible coincidence of features of metabolic syndrome the (either clinical or laboratory) or other pathological processes which could affect GGT activity, e.g. cholestasis, drug toxicity. Analysis of reasons for pre-treatment increased GGT activity was not the aim of our work. We tried to determine some of the clinical implications of change in GGT activity during treatment. We did not take into account possible coincidence of non-alcoholic fatty liver disease (NAFLD), which is currently the most common liver disease. Because of the short duration of therapy ( $\leq 12$ weeks in $98 \%$ of the patients), we assumed that significant change of the abovementioned factors influencing GGT activity was improbable.

Secondly, although in the study group characteristics, there is scarce information on laboratory markers of liver function, only class A and B Child-Pugh patients were included. Finally, we did not include longterm observation after DAA treatment, which we are hoping to perform in the following studies.

\section{Conclusions}

Our research showed that patients who, after achieving an SVR, did not exhibit reductions in both
AFP concentration and GGT activity may have higher risk of HCC development.

Special oncological monitoring may be required in the group of patients with advanced fibrosis and normal AFP concentration before treatment.

Further studies on the dynamics of changes in GGT activity after antiviral treatment may be useful in identifying a group of patients requiring close oncological surveillance.

\section{Disclosure}

The authors declare no conflict of interest.

\section{References}

1. Polaris Observatory HCV Collaborators: Global prevalence and genotype distribution of hepatitis C virus infection in 2015: a modelling study. Lancet Gastroenterol Hepatol 2017; 2: 161176.

2. Mühlberger N, Schwarzem R, Lettmeier B, et al. HCV-related burden of disease in Europe: a systematic assessment of incidence, prevalence, morbidity, and mortality. BMC Public Health 2009; 9: 34.

3. Halota W, Flisiak R, Juszczyk J, et al. Recommendations of the Polish Group of HCV Experts on the treatment of hepatitis C in 2018. Hepatologia 2018. Available from http:// www.pasl.pl/wp-content/uploads/2015/01/Hep-2018-zawarto $\%$ C $5 \% 9 B \% \mathrm{C} 4 \% 87 . p d f$

4. Alberti A, Chemello L, Benvegnu L. Natural history of hepatitis C. J Hepatol 1999; 31 Suppl 1: 17-24.

5. Li DK, Chung RT. Impact of hepatitis $C$ virus eradication on hepatocellular carcinogenesis. Cancer 2015; 121: 2874-2882.

6. Ferlay J, Soerjomataram I, Dikshit R, et al. Cancer incidence and mortality worldwide: sources, methods and major patterns in GLOBOCAN 2012. Int J Cancer 2015; 136: E359-E386.

7. Fattovich G, Stroffolini T, Zagni I, et al. Hepatocellular carcinoma in cirrhosis: Incidence and risk factors. Gastroenterology 2004; 127 (5 Suppl 1): S35-50.

8. Morgan RL, Baack B, Smith BD, et al. Eradication of hepatitis $\mathrm{C}$ virus infection and the development of hepatocellular carcinoma: a meta-analysis of observational studies. Ann Intern Med 2013; 158: 329-337.

9. Hayes CN, Zhang P, Zhang Y, et al. Molecular mechanisms of hepatocarcinogenesis following sustained virological response in patients with chronic hepatitis $\mathrm{C}$ virus infection. Viruses 2018; 10: 531 .

10. Flisiak R, Janczewska E, Łucejko M, et al. Durability of virologic response, risk of de novo hepatocellular carcinoma, liver function and stiffness two years after treatment with Ombitasvir/Paritaprevir/Ritonavir \pm Dasabuvir \pm Ribavirin in the AMBER real-world experience study. J Viral Hepat 2018; 25: 1298-1305.

11. Bloomer JR, Waldman TA, McIntire KR, et al. Alpha-fetoprotein in nonneoplastic hepatic disorders. JAMA 1975; 233: 38-41.

12. Di Bisceglie AM, Sterling RK, Chung RT, et al. Serum alpha-fetoprotein levels in patients with advanced hepatitis C: results from the HALT-C Trial. J Hepatol 2005; 43: 434-441.

13. Lu SN, Wang JH, Liu SL, et al. Thrombocytopenia as a surrogate for cirrhosis and a marker for the identification of patients at highrisk for hepatocellular carcinoma. Cancer 2006; 107: 2212-2222. 
14. Oze T, Hiramatsu N, Yakushijin T, et al. Osaka Liver Forum. Post-treatment levels of a-fetoprotein predict incidence of hepatocellular carcinoma after interferon therapy. Clin Gastroenterol Hepatol 2014; 12: 1186-1195.

15. Osaki Y, Ueda Y, Marusawa H, et al. Decrease in alpha-fetoprotein levels predicts reduced incidence of hepatocellular carcinoma in patients with hepatitis $C$ virus infection receiving interferon therapy: a single center study. J Gastroenterol 2012; 47: 444-451.

16. Silva IS, Ferraz ML, Perez RM, et al. Role of gamma-glutamyl transferase activity in patients with chronic hepatitis $\mathrm{C}$ virus infection. J Gastroenterol Hepatol 2004; 19: 314-318.

17. Everhart JE, Wright EC. Association of gamma-glutamyl transferase (GGT) activity with treatment and clinical outcomes in chronic hepatitis C (HCV). Hepatology 2013; 57: 1725-1733.

18. Mandorfer M, Reiberger T, Payer BA, et al. Revisiting predictors of virologic response to PEGIFN + RBV therapy in HIV-/ HCV-coinfected patients: the role of metabolic factors and elevated GGT levels. J Viral Hepat 2014; 21: 33-41.

19. Bergmann JF, Vrolijk JM, van der Schaar P, et al. Gamma-glutamyltransferase and rapid virological response as predictors of successful treatment with experimental or standard peginterferon-alpha-2b in chronic hepatitis $\mathrm{C}$ non-responders. Liver Int 2007; 27: 1217-1225.

20. Villela-Nogueira CA, Perez RM, de Segadas Soares JA, et al. Gamma-glutamyl transferase (GGT) as an independent predictive factor of sustained virologic response in patients with hepatitis C treated with interferon-alpha and ribavirin. J Clin Gastroenterol 2005; 39: 728-730.

21. Huang CF, Yeh ML, Tsai PC, et al. Baseline gamma-glutamyl transferase levels strongly correlate with hepatocellular carcinoma development in non-cirrhotic patients with successful hepatitis C virus eradication. J Hepatol 2014; 61: 67-74.

22. De Mello J, Struthers L, Turner R, et al. Multivariate analyses as aids to diagnosis and assessment of prognosis in gastrointestinal cancer. Br J Cancer 1983; 48: 341-348.

23. Van Hemelrijck M, Jassem W, Waldiuss G, et al. Gamma-glutamyltransferase and risk of cancer in a cohort of 545,460 persons the Swedish AMORIS study. Eur J Cancer 2011; 47: 2033-2041.

24. Announcement of the Minister of Health of 24 June 2015 on the list of reimbursed drugs, food products for particular nutritional uses and medical devices. Annex B.71. Treatment of chronic hepatitis $C$ with interferon-free therapy. Available from: http:// dziennikmz.mz.gov.pl/api/DUM_MZ/2015/27/journal/2112

25. Teegen EM, Globke B, Schott E, et al. A closing chapter: hepatitis c genotype 3 elimination in liver transplant; sofosbuvir/ daclatasvir in a hard-to-treat population. Exp Clin Transplant 2018; 16: 61-67.

26. Lleo A, Aglitti A, Aghemo A, et al. Predictors of hepatocellular carcinoma in HCV cirrhotic patients treated with direct acting antivirals. Dig Liver Dis 2019; 51: 310-317.

27. Watanabe T, Tokumoto Y, Joko K, et al. Predictors of hepatocellular carcinoma occurrence after direct-acting antiviral therapy in patients with hepatitis C virus infection. Hepatol Res 2019; 49: 136-146.

28. Nguyen K, Jimenez M, Moghadam N, et al. decrease of alpha-fetoprotein in patients with cirrhosis treated with direct-acting antivirals. J Clin Transl Hepatol 2017; 5: 43-49.

29. Yang N, Li Z, Yan M, et al. Evaluation of serum alpha-fetoprotein level in chronic hepatitis C patients. Clin Lab 2019; 65: 10.7754/Clin.Lab.2018.180607.
30. Carr BI, Pancoska P, Branch RA. Significance of increased serum GGTP levels in HCC patients. Hepatogastroenterology $2010 ; 57: 869-874$. 